\title{
Early Changes in Prefrontal Activity Characterize Clinical Responders to Antidepressants
}

Ian A. Cook, M.D., Andrew F. Leuchter, M.D., Melinda Morgan, Ph.D., Elise Witte, Ph.D., William F. Stubbeman, M.D., Michelle Abrams, R.N., Susan Rosenberg, M.S.N., and Sebastian H.J. Uijtdehaage, Ph.D.

Previous studies have shown that changes in brain function precede clinical response to antidepressant medications. Here we examined quantitative EEG (QEEG) absolute and relative power and a new measure, cordance, for detecting regional changes associated with treatment response. Fiftyone adults with unipolar depression completed treatment trials using either fluoxetine or venlafaxine vs. placebo. Data were recorded at baseline and after $48 \mathrm{~h}$ and 1 week on drug or placebo. Baseline and change from baseline values were examined for specific brain regions in four subject groups (medication and placebo responders and nonresponders). No regional baseline QEEG differences were found among the groups; there also were no significant changes in theta power over time. In contrast, medication responders uniquely showed significant decreases in prefrontal cordance at $48 \mathrm{~h}$ and 1 week. Clinical differences did not emerge until after four weeks. Subjects with greater changes in cordance had the most complete 8-week responses. These findings implicate the prefrontal region in mediating response to antidepressant medications. Cordance may have clinical applicability as a leading indicator of individual response.

[Neuropsychopharmacology 27:120-131, 2002] (C) 2002 American College of Neuropsychopharmacology. Published by Elsevier Science Inc.
KEY WORDS: Depression; Antidepressant response; Prefrontal; EEG; Cordance; Response prediction

Clinicians long have observed a lag time of several weeks between the initiation of antidepressant treatment and clinical response for many patients (Hyman and Nestler 1996; Katz et al. 1996). Some individuals do have early symptom-

From the Quantitative EEG Laboratory, Neuropsychiatric Institute and Hospital, and Department of Psychiatry and Biobehavioral Sciences, UCLA School of Medicine, University of California, Los Angeles, CA.

Address correspondence to: Dr. I.A. Cook, UCLA NPI\&H, 760 Westwood Plaza, Los Angeles, CA 90024-1759. Tel.: (310) 825-0304; Fax: (310) 825-7642; E-mail: icook@ucla.edu

Received August 20, 2001; revised January 9, 2002; accepted January 16, 2002.

Online publication: $1 / 22 / 02$ at www.acnp.org/citations/ Npp012202231. atic improvement, and this has been reported to predict further improvement over the next several weeks (Nierenberg et al. 1995). Reports have suggested that some physiologic changes are seen shortly after initiation of treatment (Sulser 1989; Beck 1995; Dahmen et al. 1997). No clinically practical physiologic predictor of treatment response has yet been identified with these techniques, however, and the relationship of early physiologic changes to eventual clinical outcome remains incompletely understood.

Quantitative electroencephalography (QEEG) has been used as a physiologic measure in efforts to address these questions. Prior work with "pharmaco-EEG" techniques has shown that the administration of antidepressant compounds yields reproducible changes in EEG activity in healthy control subjects within a few hours of dosing (Saletu et al. 1982, 1983, 1986, 1987, 1987; Saletu and Grunberger 1985, 1988; Sannita et al. 1983; Sannita 1990; 
Itil et al. 1984; Herrmann et al. 1991; Luthringer et al. 1996). The relationship of these immediate EEG changes in control subjects to eventual clinical response in a depressed population is unclear. Other QEEG work with depressed subjects has found that changes from baseline in theta power early in the course of treatment may characterize groups of depressed patients who are more likely to respond to antidepressant treatment (Ulrich et al. 1994). Unfortunately, the overlap in the value of these changes between responder and nonresponder groups precluded the use of this measure in response prediction for individual subjects, and prior research did not indicate how to relate changes in theta power to other measures of regional brain activity (e.g., regional cerebral blood flow or metabolism).

We previously have shown that absolute and relative power are complementary measures of brain activity (Leuchter et al. 1993). A relatively new QEEG measure, "cordance," combines information from both absolute and relative power measures (Leuchter et al. 1994a, 1994b, 1999). The algorithm yields two indicators: a categorical value ("concordant" or "discordant" state) and a numerical value for each electrode. In an earlier report with the categorical measure (Cook et al. 1999), we observed that depressed subjects exhibiting the concordant state prior to treatment had better treatment outcomes when treated with fluoxetine than did subjects with the discordant state. In this report, we use the num*erical values of cordance, because they allow examination of changes in regional brain activity with treatment. In validation against data collected simultaneously with $\left[\mathrm{H}_{2}{ }^{15} \mathrm{O}\right]$-positron emission tomography (PET), cordance values in the theta frequency band $(4-8 \mathrm{~Hz})$ were found to be positively correlated with cortical perfusion, and this correlation was stronger than that between perfusion and either absolute or relative theta power (Leuchter et al. 1999). The correlation of cordance with regional cortical perfusion provides a physiologic basis for interpreting this measure.

In a series of depressed subjects receiving open-label treatment, we previously have shown that cordance detects changes in prefrontal activity as early as after three days of treatment in patients who will later show clinical response to antidepressant medication (Leuchter et al. 1997; Cook et al. 1998a; Cook and Leuchter 2001). We conducted the current study to determine if QEEG power or cordance measures could reliably detect these changes in brain activity under the rigorous conditions of randomized, double-blind, placebo-controlled clinical trials. We hypothesized that prefrontal cordance values would change with treatment in responders, and that nonresponders would not show this change. In an exploratory analysis, we sought to determine whether other brain regions also implicated in mood disorders would also show changes with this measure during response. As an additional exploratory analysis, we examined the relationship between the degree of early physiologic change and the magnitude of final clinical response. Finally, we sought to determine if these early changes were specific to drug-mediated response or if they were also seen in response to placebo.

\section{METHODS AND SUBJECTS}

\section{Subjects}

We studied adults diagnosed with a major depressive episode (MDE) who were subjects in one of two separate, double-blind, randomized treatment trials conducted in our laboratory over a 24 month period: one group of subjects received fluoxetine or placebo, while subjects in the other group received venlafaxine or placebo. All subjects were free of psychotropic medication for at least two weeks prior to enrollment. All were outpatients with uni-

Table 1. Inclusion and Exclusion Criteria

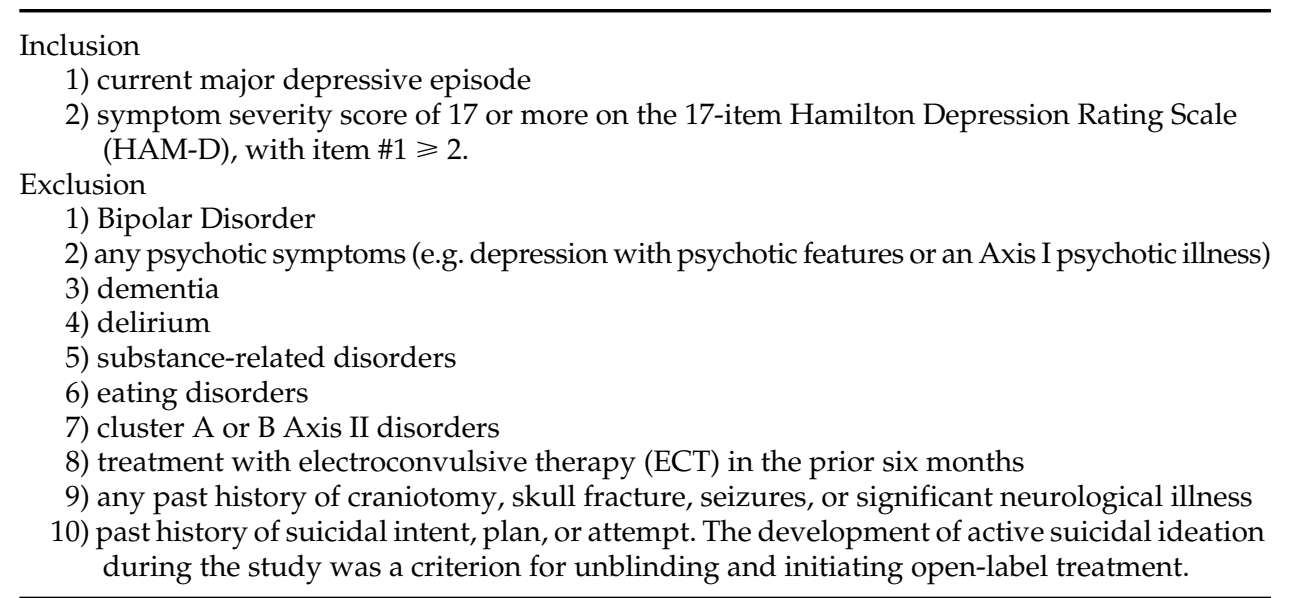


polar MDE, with diagnoses determined using a structured interview for DSM-IV (First et al. 1994), and with scores of 16 or more on the Hamilton Depression Rating Scale (HAM-D) at intake. No additional psychotropic medications were permitted during the trials (e.g., no sedative or hypnotic medications). Recruitment mechanisms as well as inclusion and exclusion criteria were identical for both protocols (Table 1). In accordance with principles of the Helsinki Declaration of 1975, both protocols were reviewed and approved by the UCLA Institutional Review Board, and informed consent to participate in this research was obtained from all subjects. Subject confidentiality was maintained for all subjects.

A total of 51 subjects completed the protocols and were examined for the present report ( 24 from the fluoxetine vs. placebo trial, and 27 from the venlafaxine vs. placebo trial). Data describing these subjects are presented in Table 2. The individuals in the fluoxetine study were the subject of our previous report on baseline characteristics (Cook et al. 1999) but had not been previously examined for early changes in regional brain activity during the course of treatment. Groups were comparable at pretreatment baseline on demographic and clinical parameters. We defined clinical response as reduction in final depression severity to a HAM-D score of 10 points or less. The two studies showed comparable drop-out rates, as well as response rates to medication and placebo. Because of the high degree of comparability of the subjects from two trials, data were pooled for the present analyses.

\section{Experimental Procedures}

Design of Treatment Trials. Subjects first entered a 1-week, single-blind placebo lead-in phase. Subjects who failed to meet study inclusion criteria at the end of lead-in (e.g., because of intolerable side effects to placebo, or a strong placebo response (no longer meeting inclusion criteria)) were removed from the protocol and were referred for open-label treatment. Subjects eligible to continue in the protocol entered a double-blind phase and were randomized to receive eight weeks of either placebo or active medication (fluoxetine $20 \mathrm{mg}$ po QD in the first protocol or venlafaxine $150 \mathrm{mg}$ po QD in the second) dispensed in identical capsules. Subjects receiving fluoxetine were given $20 \mathrm{mg} / \mathrm{d}$ and continued at that dose for the eight weeks; subjects receiving venlafaxine began at $37.5 \mathrm{mg} / \mathrm{d}$, increased over a week to $150 \mathrm{mg} / \mathrm{d}$, and then continued at that dose for the remaining seven weeks. To preserve blinding, placebo "dose" was escalated in the second protocol.

All subjects received brief sessions of supportive psychotherapy during the blinded phase of the study, in order to address safety concerns about dispensing placebo alone to patients with significant depression (15-25 min of unstructured counseling and assistance in problem solving by a research nurse at the follow-up visits). Follow-up visits for symptom/side effect monitoring and for the supportive therapy took place at two days and at weekly intervals thereafter after the start of the double-blind phase of the study. Symptoms were monitored with a focused clinical interview, clinician rating scales (e.g., HAMD), and self-rating scales. At the end of the 8-week doubleblind phase, the blinding was broken and HAM-D scores were used to categorize subjects as responders or nonresponders. Subjects who had received placebo were eligible to begin open-label treatment; subjects who had received medication were eligible for continued pharmacotherapy.

\section{QEEG Techniques}

Data Acquisition. QEEG recordings were obtained: (1) at pretreatment baseline prior to randomization; (2) at $48 \mathrm{~h}$ (after two doses of drug or placebo); and (3) after one week on medication or placebo, as shown in Figure 1. Recordings were made with the QND System (Neurodata, Inc., Pasadena, CA), using procedures employed in our previ-

Table 2. Characteristics of Subjects

\begin{tabular}{lcccccc}
\hline & $\begin{array}{c}\text { Fluoxetine } \\
\text { study } \\
\mathbf{n = 2 4}\end{array}$ & $\begin{array}{c}\text { Venlafaxine } \\
\text { Study } \\
\mathbf{n}=\mathbf{2 7}\end{array}$ & $\begin{array}{c}\mathbf{M}-\mathbf{R} \\
\mathbf{n}=\mathbf{1 3}\end{array}$ & $\begin{array}{c}\mathbf{M}-\mathbf{N R} \\
\mathbf{n}=\mathbf{1 2}\end{array}$ & $\begin{array}{c}\text { P-R } \\
\mathbf{n}=\mathbf{1 0}\end{array}$ & $\begin{array}{c}\text { P-NR } \\
\mathbf{n}=\mathbf{1 6}\end{array}$ \\
\hline Age & $40.3 \pm 11.5$ & $42.6 \pm 12.5$ & $41.2 \pm 11.7$ & $42.3 \pm 13.2$ & $38.2 \pm 15.2$ & $43.2 \pm 9.6$ \\
Gender ratio (M:F) & $8: 16$ & $11: 16$ & $3: 10$ & $4: 8$ & $6: 4$ & $6: 10$ \\
Family History (y:n) & $11: 13$ & $20: 7$ & $7: 6$ & $7: 5$ & $5: 5$ & $12: 4$ \\
Baseline HAM-D & $21.8 \pm 4.2$ & $22.4 \pm 3.1$ & $21.5 \pm 3.0$ & $23.3 \pm 4.3$ & $20.7 \pm 3.3$ & $22.6 \pm 3.6$ \\
Baseline BDI & $27.0 \pm 7.3$ & $26.8 \pm 8.3$ & $28.1 \pm 8.0$ & $26.7 \pm 7.2$ & $24.0 \pm 10.0$ & $27.8 \pm 6.5$ \\
Baseline HAM-A & $20.0 \pm 5.9$ & $21.9 \pm 5.6$ & $21.1 \pm 4.6$ & $21.2 \pm 6.0$ & $19.0 \pm 7.0$ & $21.9 \pm 5.9$ \\
Baseline MADRS & $33.0 \pm 4.5$ & $32.9 \pm 4.3$ & $32.8 \pm 3.7$ & $33.7 \pm 5.5$ & $31.3 \pm 3.3$ & $35.6 \pm 4.6$ \\
\hline
\end{tabular}

Study groups (fluoxetine and venlafaxine group, as well as the four response cells) were comparable on all clinical severity measures and for age and gender distribution. No differences were found for personal history of lifetime depressive episodes (categories: 1, 2-3, 4 or more, $\chi^{2}$ n.s.). Differences between medication groups were present for family history of depression $\left(\chi^{2}=4.25, \mathrm{df}=1, \mathrm{p}=.04\right) .13$ of the 24 subjects in the fluoxetine study group received active medication; 12 of the 27 subjects received venlafaxine in the other group. HAM-D: Hamilton Depression Rating Scale; HAM-A: Hamilton Anxiety Rating Scale; BDI: Beck Depression Inventory; MADRS: Montgomery Asberg Depression Rating Scale. 


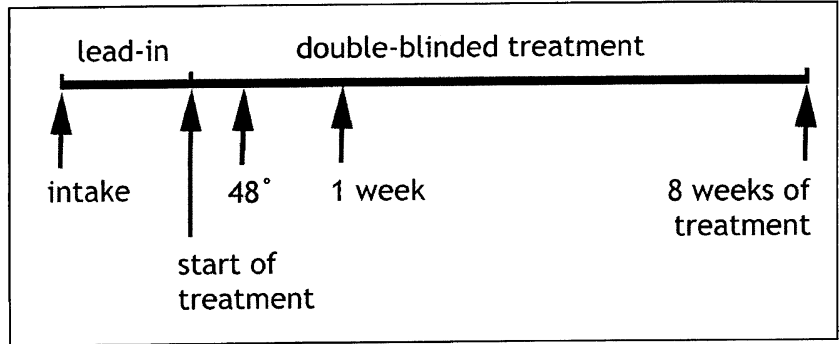

Figure 1. Experimental protocol timeline. Subjects were assessed and enrolled at "intake," had the pretreatment baseline EEG recorded at that time, and then participated in a 1-week, single-blind placebo lead-in phase. Randomization to treatment modality (active medication or placebo) took place at the time marked "start of treatment." Another EEG was recorded after $48 \mathrm{~h}$ of treatment and again at 1 week. Clinical assessment to determine outcome (responder vs. nonresponder) took place after eight weeks of treatment (9 weeks in study altogether). Subjects were monitored weekly during the double-blind treatment (arrows not shown) for clinical changes and adverse reactions.

ous reports and summarized here. Subjects were instructed to rest in the eyes-closed, maximally alert state, in a quiet room with subdued lighting. The technicians monitored the QEEG data during the recording and re-alerted the subjects every $30-45 \mathrm{~s}$ as needed to avoid drowsiness. Electrodes were placed with an electrode cap (ElectroCap, Eaton, $\mathrm{OH})$ using 35 recording electrodes distributed across the head according to the International 10-20 System arrangement (Figure 2). Data were collected using a Pz referential montage and were digitized at 256 samples/chan$\mathrm{nel} / \mathrm{sec}$ by the QND system (bandpass filtered $0.3-70 \mathrm{~Hz}$ ).

Each EEG recording was reviewed by a technician who was blinded to subject identity, treatment condition, and clinical status; the first 20-32 s of artifact-free data were selected to be processed. A second technician reviewed these selections for accuracy. These selections were then processed using a fast Fourier transform to obtain absolute and relative power values in four frequency bands $(0.5-4 \mathrm{~Hz}, 4-8 \mathrm{~Hz}, 8-12 \mathrm{~Hz}$, and $12-20 \mathrm{~Hz})$. "Absolute power" describes the amount of power in a frequency band at a given electrode (measured in $\mu V^{2}$ ), and "relative power" is the percentage (\%) of power contained in a frequency band, relative to the total power across the entire spectrum $(0.5-20 \mathrm{~Hz})$ computed separately for each electrode. The recording data were reformatted offline to compute linked-ear-reference absolute and relative power values; these values were used in the regional measure analyses described below. QEEG data also were reformatted to bipolar channel pairs and processed further to yield cordance values (below).

\section{Cordance Calculations}

For each recording site in each of the four bands, cordance values were calculated using an algorithm that has been detailed elsewhere (Leuchter et al. 1999) and may be summarized as follows. Cordance is computed by a normal-

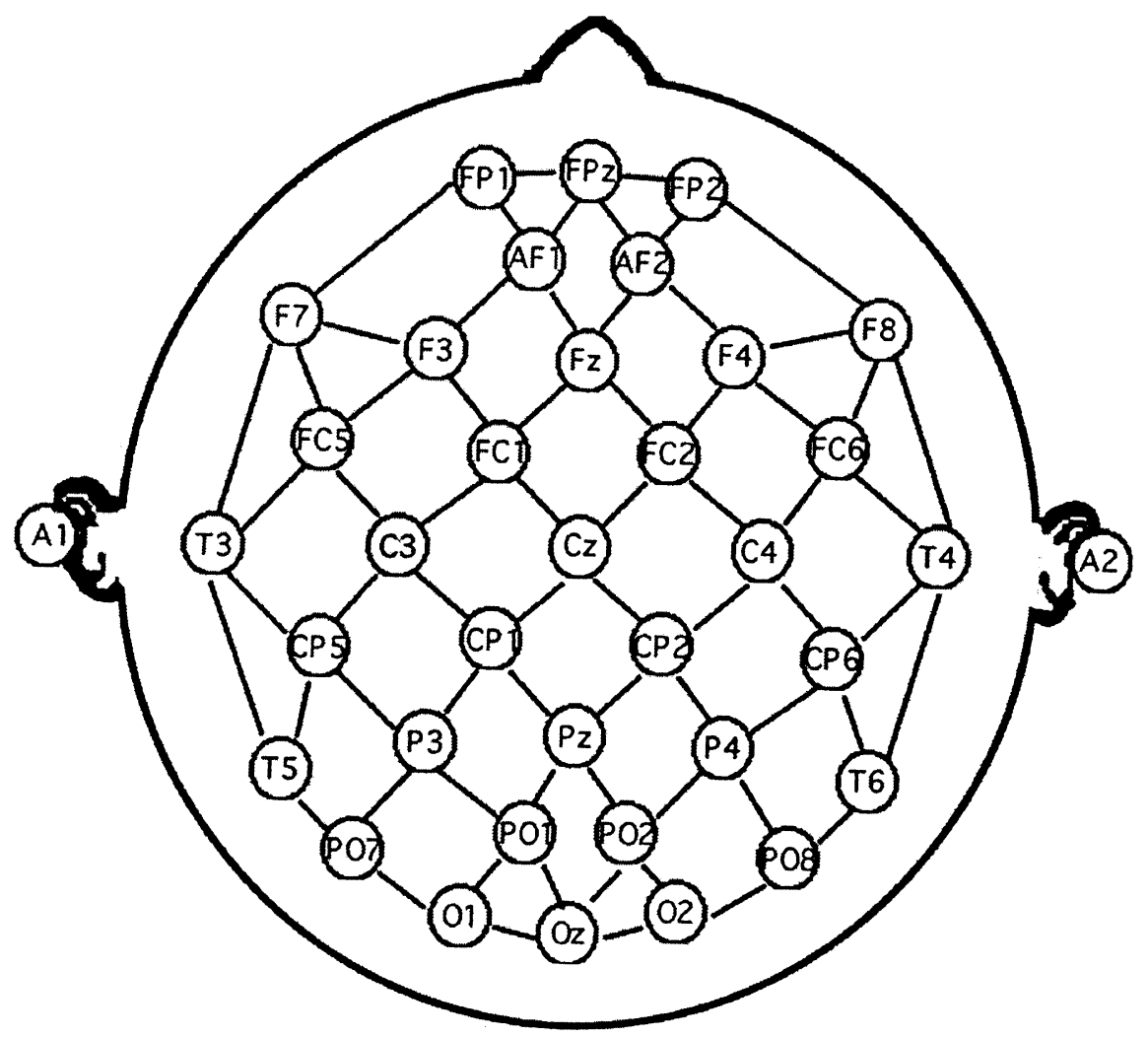

Figure 2. Electrode montage. The 35 scalp electrodes from the extended International 10-20 system. Line segments denote bipolar channels used in the reattributional montage. Electrodes included in the calculation of average prefrontal cordance are Fp1, Fp2, Fpz (after Cook et al. 1998b). 
ization and integration of absolute and relative power values from all electrode sites for a given EEG recording; cordance values are calculated in three steps. First, EEG power values are computed using a re-attributional electrode montage in which power values from pairs of electrodes that share a common electrode are averaged together to yield the re-attributed power (Figure 2) (Cook et al. 1998b). For example, to determine a power value for the brain region underlying the F4 electrode, one first computes power spectra for the channels that include the F4 electrode (i.e., F4-F8, F4-AF2, F4-FC2, and F4-FC6, in Figure 2), and then averages the absolute power values from those channels to obtain the reattributed power for the F4 electrode. This is somewhat similar to the single source method of Hjorth $(1970,1975)$, but cordance recombines the power values whereas Hjorth's method recombines voltage signals by averaging signal amplitudes from pairs of electrodes. The Hjorth method is preferred under many experimental designs, particular when the source of a signal is the question of interest (e.g., seizure focus); the re-attributional montage provides a higher association between QEEG measures and regional cortical perfusion than does the Hjorth method (Cook et al. 1998b) and so offers an advantage for testing our specific hypotheses. Relative power is calculated in the conventional manner, as the percentage of power in each band, relative to the total spectrum considered (here, $0.5 \mathrm{~Hz}$ to $20 \mathrm{~Hz}$ ) (cf. Leuchter et al. 1993).

Second, these absolute and relative power values for each individual EEG recording are normalized across electrode sites, using a z-transformation statistic for each electrode site $s$ in each frequency band $f$ (yielding $\mathrm{A}_{\text {norm(s,f) }}$ and $R_{\text {norm }(s, f)}$ respectively). It should be noted that these $z$-scores are based on the average power in each band for all electrodes within a given QEEG recording, and are not z-scores referenced to some normative population (e.g., as in the "neurometrics" approach, (cf., John et al. 1988)). The normalization process places absolute and relative power values into a common unit (standard deviation or $\mathrm{z}$ score units) which allows them to be combined.

Third, the cordance values are formed by summing the $\mathrm{z}$-scores for normalized absolute and relative power $\left(Z_{(s, f)}=A_{\text {norm }(s, f)}+R_{\text {norm }(s, f)}\right.$, for each electrode site and in each frequency band). Cordance values have been shown to have higher correlations with regional cerebral blood flow than absolute or relative power alone (Leuchter et al. 1999), and thus this combination measure can be placed in context with prior work in depression that employed functional measures of brain activity such as PET scan data.

\section{Regional Measures}

To study changes in regional brain activity, the values from electrodes overlying specific neuroanatomic regions were averaged together. We examined regions that have been implicated by prior work for involvement in mood disorders. The regional groupings employed were (1) prefrontal (Fp1, Fp2, Fpz electrodes); (2) central (overlying the cingulate gyrus) (FC1, FC2, Cz electrodes); (3) left temporal (T3, T5 electrodes); and (4) right temporal (T5, T6 electrodes). These regional measures were calculated for absolute power, relative power, and cordance values. In order to avoid spurious findings from comparing a large number of potential variables, analyses were limited to the theta band, since it has previously been shown to be sensitive to changes in activity in depression (Ulrich et al. 1988; Leuchter et al. 1997; Cook et al. 1998a; Cook and Leuchter 2001).

\section{Data Analysis}

The subjects were examined in four groups: medication responder ("M-R") and nonresponder ("M-NR") groups, and placebo responder ("P-R") and nonresponder ("P$\left.N R^{\prime \prime}\right)$ groups. A final HAM-D score of $\leqslant 10$ at the end of the 8-week double-blind phase was used to define the responders (Cook et al. 1999). As noted earlier, subjects from the fluoxetine and venlafaxine trials did not differ significantly in response rates or demographic parameters and thus were pooled for these analyses.

Continuous variable data were analyzed with analysis of variance (ANOVA), $t$-tests, and regression analyses; discrete variables with limited range (e.g., number of previous episodes of depression) were compared using nonparametric tests; categorical data were examined using the $\chi^{2}$ statistic (SPSS, Inc., Chicago, IL). Cordance, absolute power and relative power were examined for group differences in the prefrontal, central, right temporal, and left temporal regions using ANOVA. To evaluate changes over time, repeated measures analysis of variance was used in each region to examine changes in these physiologic measures, employing treatment response (M-R, M-NR, P-R, P-NR) as the between-group factor and time ( $48 \mathrm{~h}, 1$ week) as the within-group factor. The full factorial model tested the response effect, the time effect, and the response by time interaction. Post hoc pairwise comparisons were performed where significant effects were observed with ANOVA, using the Bonferroni correction to avoid Type 1 errors from multiple comparisons.

In addition to the group analyses, the ability of cordance to predict individual response was tested by classifying subjects on the basis of change in cordance at each time point. Chi-square tests were used to test the hypothesis of independence of the classification variable and the response variable.

\section{RESULTS}

\section{Clinical Responses}

Thirteen of 25 subjects (52\%) responded to medication: seven of 13 subjects $(54 \%)$ responded to treatment with 
fluoxetine, and six of $12(50 \%)$ responded to venlafaxine. Response rates to medication between the two studies were not statistically different. The placebo response rate was $38 \%$ (10 of 26). A majority of subjects in both responder groups had sustained decreases in depression rating scores (7/13 M-R and 7/10 P-R). Responders were significantly different from nonresponders on final HAM-D $\left(\left(\mathrm{F}_{1,49}=121.4, p<.0001\right)\right.$. Both responder groups had comparable clinical outcomes (final HAM-D $6.0 \pm 3.1$ for $\mathrm{M}-\mathrm{R}$ and $6.1 \pm 3.4$ for P-R); both nonresponder groups also showed equivalent clinical endpoints (final HAM-D $18.4 \pm 4.6$ for M-NR and $17.9 \pm$ 4.5 for P-NR).

\section{Regional Differences in Power and Cordance at Baseline and Changes over Time}

Absolute power, relative power, and cordance were examined for group differences (M-R, M-NR, P-R, P-NR) for differences at pretreatment baseline in any of the regions (prefrontal, central, temporal) using analysis of variance (ANOVA). There were no statistically significant differences among the response groups for any region for absolute power, relative power, or cordance (Table 3).

Because there were no baseline differences, we next examined changes over time in the three QEEG measures by creating variables to reflect the change from baseline at the two time points ( $48 \mathrm{~h}, 1$ week). Repeated measures analysis of variance was used in each region to examine changes in these physiologic measures. Neither absolute nor relative power measures showed any significant differences.

In contrast, the group effect was significant for cordance in the ANOVA. Pairwise comparisons using the Bonferroni adjustment revealed a significant group effect exclusively in the prefrontal region in the medication responder group (Figure 3, Table 4), with a trend- level finding at $48 \mathrm{~h}\left(\mathrm{~F}_{3,46}=2.37, p=.083\right)$ and significant group differences at one week $\left(\mathrm{F}_{3,47}=4.11, p=\right.$ .011) where M-R was significantly different from M-NR, $\mathrm{P}-\mathrm{R}$, and P-NR.

Examining the changes independently for each group, the M-R group exhibited a significant decrease in prefrontal cordance from baseline values at $48 \mathrm{~h}$ (2-tailed $\mathrm{t}=2.78, \mathrm{df}=12, p=.017)$ and one week $(\mathrm{t}=$ $2.71, \mathrm{df}=12, p=.019$ ) (Figure 3, Table 4). The topography of these changes is shown in Figure 4. There were no significant decreases seen for M-NR, P-R, or P-NR subjects at $48 \mathrm{~h}$ or 1 week (Table 4 ). The decrease in cordance in the M-R subjects was not significantly different between fluoxetine and venlafaxine trials.

\section{Relationship of Change in Cordance to Degree of Response}

Given this finding of decrease in prefrontal cordance in the $\mathrm{M}-\mathrm{R}$ subjects, we used a series of linear regression models to examine the relationship between magnitude of cordance change and degree of clinical response for all subjects receiving medication. Final HAM-D score was significantly predicted by change in cordance at one week $(\mathrm{r}=0.51, \mathrm{df}=23, p=.002)$, with greater physiologic change predicting better clinical response; the relationship was not significant at $48 \mathrm{~h}(\mathrm{r}=0.22$, df $=23, p=.29$ ) (Figure 5). Similar relationships were found for change in HAM-D from baseline to final evaluation. Regression models were also performed to test whether change in cordance was associated with clinical severity at other time points, specifically to evaluate if the physiologic changes were present prior to clinical changes or if the physiologic and clinical measures changed concurrently. HAM-D score at $48 \mathrm{~h}$ was not related to change in cordance at $48 \mathrm{~h}$, and HAM-D at one week was not related to change in cordance at either $48 \mathrm{~h}$ or 1 week. To evaluate other potential confounding fac-

Table 3. Comparison of Baseline Power and Cordance in Responders and Nonresponders

\begin{tabular}{llcccc}
\hline Region & \multicolumn{1}{c}{ Measure } & M-R & M-NR & P-R & P-NR \\
\hline Frontal & absolute power & $22.79 \pm 18.06$ & $22.56 \pm 12.28$ & $26.67 \pm 21.04$ & $25.69 \pm 15.64$ \\
& relative power & $16.15 \pm 4.24$ & $17.80 \pm 9.30$ & $16.51 \pm 8.36$ & $16.15 \pm 4.24$ \\
& cordance & $-0.25 \pm 1.03$ & $-0.62 \pm 1.43$ & $-1.62 \pm 1.12$ & $-0.84 \pm 1.03$ \\
Central & absolute power & $44.14 \pm 38.59$ & $39.09 \pm 21.79$ & $53.39 \pm 43.92$ & $45.50 \pm 19.99$ \\
& relative power & $22.57 \pm 5.63$ & $21.43 \pm 9.07$ & $20.87 \pm 9.85$ & $21.19 \pm 6.06$ \\
& cordance & $0.77 \pm 1.24$ & $0.32 \pm 1.07$ & $0.57 \pm 1.17$ & $0.52 \pm 1.46$ \\
Left & absolute power & $22.39 \pm 25.00$ & $17.20 \pm .08$ & $34.70 \pm 39.88$ & $20.88 \pm 13.88$ \\
Temporal & relative power & $17.03 \pm 4.100$ & $16.67 \pm 8.18$ & $18.50 \pm 9.37$ & $17.03 \pm 4.00$ \\
& cordance & $0.26 \pm 1.08$ & $0.89 \pm 1.20$ & $1.09 \pm 0.91$ & $0.87 \pm 1.23$ \\
Right & absolute power & $23.20 \pm 20.60$ & $18.54 \pm 12.78$ & $32.50 \pm 12.50$ & $21.17 \pm 12.44$ \\
Temporal & relative power & $17.52 \pm 4.74$ & $16.08 \pm 9.84$ & $16.99 \pm 8.47$ & $17.32 \pm 5.27$ \\
& cordance & $0.45 \pm 0.84$ & $0.51 \pm 1.28$ & $0.52 \pm 1.15$ & $0.93 \pm 1.34$ \\
\hline
\end{tabular}

Baseline absolute power $\left(\mu \mathrm{V}^{2}\right)$, relative power (\% total power), and cordance values (z-score units) for regions of interest were compared across the four subject groups (M-R, M-NR, P-R, P-NR) via ANOVA. No significant group differences were seen. 


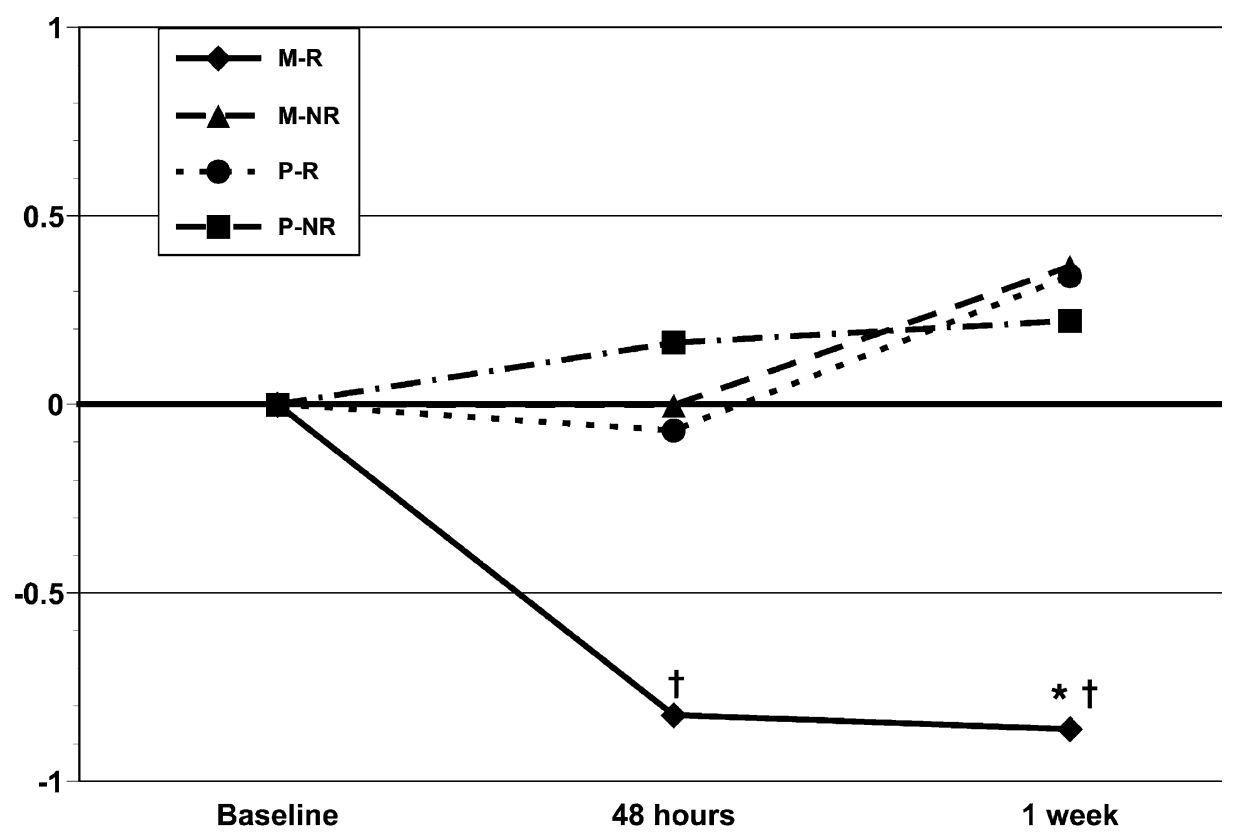

TIME ON MEDICATION / PLACEBO
Figure 3. Time course of physiologic changes in responders and nonresponders. Changes from baseline values for group average prefrontal cordance values are plotted at $48 \mathrm{~h}$ and 1 week for the four response groups, medication responder (M-R) and nonresponder (M-NR) groups and placebo responder (P-R) and nonresponder (P-NR) groups. Repeated measures ANOVA shows that the M-R group is significantly different from the other three groups at 1 week $\left(\mathrm{F}_{3,47}=4.11, p=.011 *\right)$. M-R subjects are significantly different from baseline at $48 \mathrm{~h}$ (2-tailed $\mathrm{t}=$ 2.78, $\left.\mathrm{df}=12, p=.017^{\dagger}\right)$, and one week $\left(\mathrm{t}=2.71, \mathrm{df}=12, p=.019^{\dagger}\right)$; no other groups show significant changes from their respective baseline values. tors, we also examined the relationship between baseline cordance value and final HAM-D and found that there was no significant influence of pretreatment value on outcome $(\mathrm{r}=-0.25, \mathrm{df}=23, p=.24)$

\section{Accuracy of Cordance in Predicting Clinical Outcome}

In addition to the group analyses above, we also examined the data on an individual-by-individual basis to determine the accuracy of cordance in predicting 8-week clinical outcome in response to medication. Each subject was categorized as either showing a decrease in prefrontal cordance at each time point or not showing the decrease. Using a categorization of decrease to pre- dict response and non-decrease to predict non-response, the accuracy or "test efficiency" was $64 \%$ at $48 \mathrm{~h}$ (9 of 13 M-R; 7 of $12 \mathrm{M}-\mathrm{NR} ; \chi^{2}=1.92, \mathrm{df}=1, p=.17$ ), and $72 \%$ at 1 week ( 9 of $13 \mathrm{M}-\mathrm{R} ; 9$ of $12 \mathrm{M}-\mathrm{NR} ; \chi^{2}=4.89, \mathrm{df}=1, p=$ .027 ; sensitivity $69 \%$, specificity $75 \%$, positive predictive value $75 \%$, negative predictive value $69 \%$ ).

\section{COMMENT}

Three primary findings emerge from this study. First, the response to treatment with fluoxetine or venlafaxine was characterized by an early decrease in prefrontal cordance, a change which was not present in nonresponders. This change emerged as early as after two

Table 4. Neurophysiologic and Clinical Changes in Responders and Nonresponders

\begin{tabular}{lcccr}
\hline Subject Group: & \multicolumn{1}{c}{ M-R } & M-NR & P-R & \multicolumn{1}{c}{ P-NR } \\
\hline Change in relative power at $48 \mathrm{~h}$ & $-1.00 \pm 8.33$ & $0.12 \pm 2.62$ & $2.42 \pm 6.1$ & $0.42 \pm 3.53$ \\
Change in relative power at 1 week & $-1.95 \pm 7.25$ & $-0.51 \pm 4.58$ & $2.90 \pm 4.08$ & $-0.46 \pm 4.15$ \\
Change in cordance at $48 \mathrm{~h}$ & $-0.82 \pm 1.07^{+}$ & $-0.00 \pm 1.25$ & $-0.07 \pm 1.14$ & $0.16 \pm 0.78$ \\
Change in cordance at 1 week & $-0.86 \pm 1.15^{*+}$ & $0.37 \pm 0.81$ & $0.34 \pm 0.82$ & $0.22 \pm 1.19$ \\
Change in HAM-D at 48 h & $-6.2 \pm 3.8$ & $-4.6 \pm 3.0$ & $-4.9 \pm 3.6$ & $-4.8 \pm 5.2$ \\
Change in HAM-D at 1 week & $-9.3 \pm 4.9$ & $-4.5 \pm 4.0$ & $-7.1 \pm 5.2$ & $-5.9 \pm 5.2$ \\
\hline
\end{tabular}

Change from baseline in prefrontal relative power and in cordance were determined after $48 \mathrm{~h}$ and one week of treatment with active medication (M-R, M-NR) or placebo (P-R, P-NR). For prefrontal relative power, no significant group differences were found by ANOVA in change from baseline at $48 \mathrm{~h}$ or one week. For prefrontal cordance, ANOVA revealed a trend-level finding at $48 \mathrm{~h}\left(\mathrm{~F}_{3,46}=2.37, p=.083\right)$ and significant group differences at one week $\left({ }^{*} \mathrm{~F}_{3,47}=4.11, p=.011\right)$ where M-R was significantly different from M-NR, P-R, and P-NR. For HAM-D scores, ANOVA revealed no significant changes from baseline at $48 \mathrm{~h}$ or one week (trend at one week: $\mathrm{F}_{3,47}=.023, p=.097$ ). M-R subjects are significantly different from their baseline at $48 \mathrm{~h}\left(2\right.$-tailed $\left.\mathrm{t}=2.78, \mathrm{df}=12, p=.017^{\dagger}\right)$, and one week $\left(\mathrm{t}=2.71, \mathrm{df}=12, p=.019^{\dagger}\right)$; no other groups show significant changes from their respective baseline values. 


\section{8 hours}

Medication

Responders
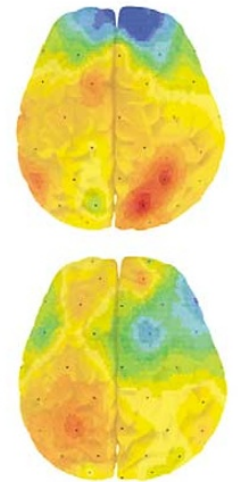

Medication

Nonresponders

\section{Placebo \\ Responders}

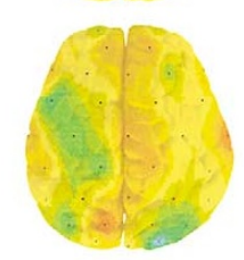

Placebo

Nonresponders

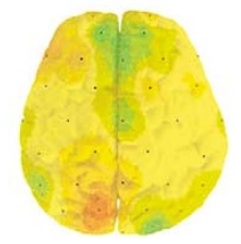

\section{1 week}
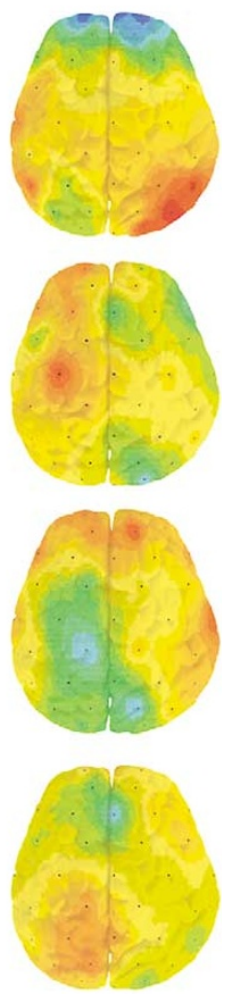

Figure 4. Topography of early physiologic changes. Cordance maps show early decreases in prefrontal cordance in medication responders. Yellow indicates no change, green-blue indicates a decrease from baseline, while orange-red indicates an increase (on a scale from -0.7 to +0.7 average change in cordance). Maps show the head as viewed from above, with the prefrontal regions at the top of each map. Decreases uniquely characterize the medication responder subjects.

doses of medication, and was specific to the prefrontal region. Predictive changes were not seen with absolute or relative power in any region. Second, we also found that the degree of the decrease in prefrontal cordance was a leading indicator of the magnitude of eventual response: greater early decreases in cordance were significantly predictive of better final outcome. Third, neither placebo responders nor placebo nonresponders exhibited these patterns. To our knowledge, these are the first data indicating that early physiologic changes in the prefrontal regions are related to the completeness of clinical response.

Prior reports often have shown changes in power spectra that were associated with the administration of antidepressants. In this study, absolute and relative power did not show a statistically significant increase in the presence of medication, and we did not find any differences in absolute or relative between responders and nonresponders. The inconsistency between our findings and the prior reports of Ulrich (1988) or Knott et al. (1996) may reflect differences in the medications

used (maprotoline and clomipramine in Ulrich's report; imipramine in Knott's study), since those tricyclic antidepressants have patterns of activity that involve more neurotransmitter systems known to have non-specific effects on EEG activity (e.g., acetylcholine). Since absolute and relative power measures individually failed to detect the early predictive changes that were seen with cordance in our dataset, there may be an advantage in combining power measures via the cordance algorithm.

In addition, the relationship of cordance values to cortical perfusion provides a context in which to compare cordance results with other methods of assessing brain activity. Our findings are consistent with some previous reports that examined frontal changes during treatment, using PET or single-photon emission computed tomography (SPECT) methods. In a series of studies using [ $\left.{ }^{133} \mathrm{Xe}\right]-S P E C T$ to examine patients undergoing electroconvulsive therapy (ECT), Sackeim and colleagues (Sackeim et al. 1994; Nobler et al. 1994) reported decreases in frontal cerebral perfusion in subjects responding to ECT; this group more recently reported similar findings in subjects treated with medications (Nobler et al. 1998). Martinot et al. (1990) and Austin et al. (1992) also reported reduced cerebral activity associated with treatment with antidepressant medications, using PET and SPECT respectively. In a relatively large sample of subjects with depression, Drevets and colleagues (Drevets and Raichle 1992; Drevets et al. 1997; Drevets 1994) reported that any decreases in perfusion during treatment were found most prominently in prefrontal cortical regions. Brody and colleagues (1999, 2001) also have reported decreases in prefrontal cortex metabolism in subjects responding to paroxetine treatment. Since cordance values in the theta band are positively correlated with cerebral perfusion, a physiologic interpretation of our QEEG results is that early neurophysiologic changes in responders to fluoxetine or venlafaxine are related to decreases in prefrontal perfusion, while nonresponders lack this change. A direct comparison of our cordance results with these prior PET and SPECT studies is limited by the different time-frames employed in the observations: changes over $4-8$ weeks of treatment for the neuroimaging studies vs. changes after $48 \mathrm{~h}$ or 1 week of treatment in the current study.

There is additional evidence from neuropsychological studies that frontal dysfunction is involved in the pathophysiology of depressive illnesses and may be related to treatment response (Drevets et al. 1999; Kalayam and Alexopoulos 1999; Dunkin et al. 2000). Frontal dysfunction and decreased perfusion in prefrontal regions may be primary abnormalities in brain activity, but could also be secondary manifestations of alterations in other brain regions which project to the prefrontal cortex. Studies are needed explicitly to address the relationship of prefrontal cortical changes to activity in other parts of the limbic system. 


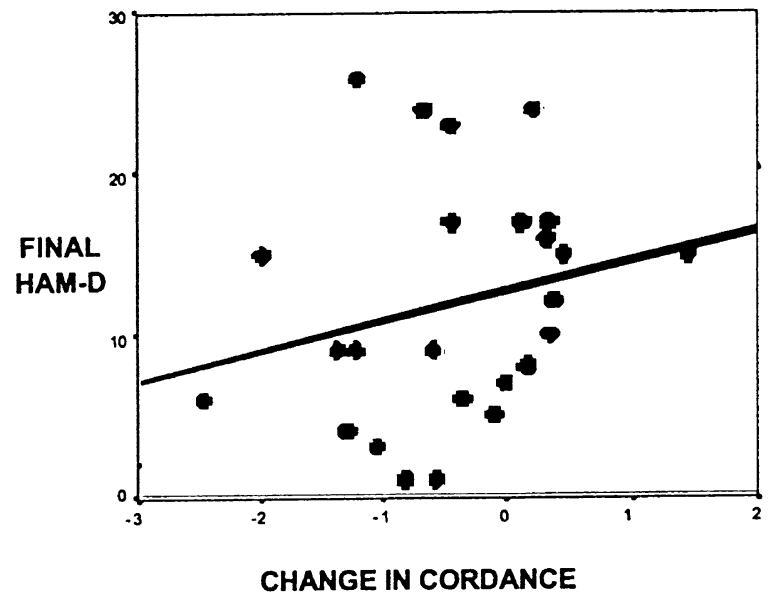

(a) 48 hours

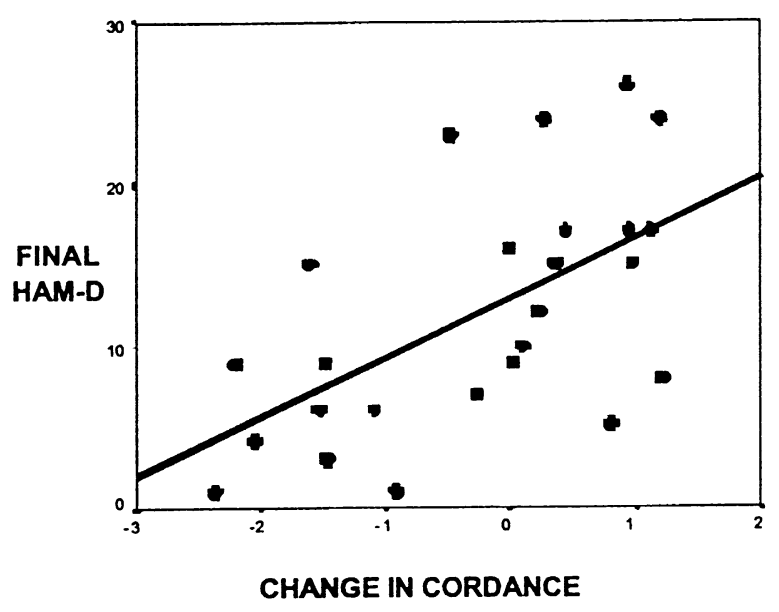

(b) 1 week

Figure 5. Relationship of early physiologic change to degree of clinical improvement. Early changes in prefrontal cordance are associated with final HAM-D clinical outcomes. Final HAM-D scores at week 8 are plotted as a function of change in cordance value at the times indicated. Linear regression models do not show a significant association of final response and change in cordance at $48 \mathrm{~h}$ (panel a: $\mathrm{r}=0.22, \mathrm{df}=23, p=.29$ ), but significant predictions of final clinical severity by change in cordance are seen at one week (panel b: $\mathrm{r}=.051, p=.002, \mathrm{df}=23$ ).

While some neuroimaging studies have reported that response is associated with regional decreases in activity (as above), others have reported increases in activity (e.g., Baxter et al. 1989; Dube et al. 1993; Bonne et al. 1996; Bonne and Krausz 1997). At least one group (Hurwitz et al. 1990) has reported no change in cerebral metabolism measured with PET. This disagreement within the neuroimaging literature may reflect differences in the techniques used to assess cerebral physiology, the particular antidepressant medication under examination, the population studied, their clinical symptoms, or study designs that lack a placebo comparison condition.

Other changes in neurophysiologic function from antidepressant medications also contribute to the context in which our findings can be considered, particularly with regard to region and timeframe. Yatham and colleagues (1999) examined changes in 5-HT receptor binding between baseline and 3-4 weeks on desipramine via PET data. They found the administration of this agent was accompanied by a decrease in binding seen most prominently in prefrontal cortex, a change which is consistent with the prefrontal topography of our cordance changes, albeit over a longer timeframe than in the current study. Receptor downregulation has been reported as part of the response to chronic antidepressant administration since the 1970s (cf. Sulser 1989), but may also be detected within several hours of the administration of a single dose of an antidepressant (Newman-Tancredi et al. 1996), suggesting the plausibility of detecting meaningful changes after only two doses. Induction of specific genes (e.g., $c$-fos) has been elicited with a single exposure to antidepressants (Dahmen et al. 1997; Beck 1995; Muck-Seler et al. 1996). Fluoxetine has also been shown directly to alter membrane currents from the 5-HT receptors with acute administration (Pitt et al. 1994; Ni and Miledi 1997); this is a distinctly different action from its classic effect of inhibiting presynaptic serotonin reuptake transporters, but could affect EEG signals. The contribution of these early changes to the clinical response in depressed humans remains to be fully understood.

Alvarez and colleagues (1999) have reported data relating acute changes in serum serotonin levels to clinical outcome. After a single dose of fluoxetine, plasma 5-HT levels had decreased from pretreatment values in those who would eventually respond (using 2 and 4 week clinical endpoints) while the eventual nonresponders showed an increase in serum 5-HT after one day on fluoxetine. They interpret their results to indicate that inhibition of 5-HT uptake is a necessary but insufficient condition for fluoxetine's antidepressant properties. Our time scale of change in brain function is comparable to what they observed with their peripheral measure.

Our finding that the magnitude of clinical response is proportional to the extent of early physiologic change is intriguing. Examining physiologic activity and clinical response as related continuous measures can serve as a complementary approach to much of the prior work with EEG or with neuroimaging, where categorical analyses are more common (e.g. Ulrich et al. 1998; Knott et al. 1996; Sackeim et al. 1994; Nobler et al. 1994; Baxter et al. 1989; Dube et al. 1993; Bonne et al. 1996). The subjects 
with the best clinical responses in our study tended to show larger physiologic changes. One interpretation of our finding is that subjects who exhibit a higher degree of physiologic response to the introduction of a medication will later exhibit a larger clinical effect, while subjects who do not show much physiologic sensitivity to the medication will not experience much clinical benefit.

We view these findings as preliminary and in need of further examination with a larger subject pool. In evaluating any test potential clinical application, the primary questions of reliability, sensitivity, and specificity need to be addressed in an adequately large sample for independent replication. In addition, questions of time, effort, cost, specialized technical skills, and patient friendliness are pragmatic issues that also must be addressed in future investigations. As noted in the Acknowledgments, software to perform the cordance algorithm is available at no cost to investigators for research purposes, to address questions regarding the generalizability of these findings and the potential for more widespread application of the measure.

It is interesting that the changes in prefrontal cordance were seen in responders to both fluoxetine and venlafaxine; fluoxetine has a greater effect on serotonin than on norepinephrine reuptake, while venlafaxine's mechanism of action involves both neurotransmitter systems (Frazer 2001) although the serotonergic effects may be most important at the starting dose used in this project. The shared prefrontal change may reflect a common-pathway role for the prefrontal regions in the clinical response, whether this arises via serotonergic or mixed neurotransmitter influences for any one individual. These results suggest that it may be possible to use cordance to make early, multiple observations of brain function in depressed subjects to predict medication effectiveness. Additional confirmation of the stability and reliability of the cordance measure is necessary before it can be more broadly applied.

\section{ACKNOWLEDGMENTS}

This work was supported by a Young Investigator Award (Rio Hondo Investigator) to Dr. Cook from the National Alliance for Research in Schizophrenia and Depression (NARSAD), by Career Development Award K08-MH01483 from the National Institute of Mental Health to Dr. Cook, and by grant R01-MH40705 and Research Scientist Development Award K02-MH01165 from the National Institute of Mental Health to Dr. Leuchter. Dr. Stubbeman and Dr. Morgan were supported by NIMH Fellowship grant T32-MH17140. We also wish to acknowledge the grant support of Eli Lilly and Company, Inc. and Wyeth-Ayerst Laboratories, Inc. in conducting this work. Additionally, we would like to acknowledge the assistance of Mr. Steven David and Ms. Koren Hanson in data management, and of Ms. Kelly Nielson and Mr. David Barrad in manuscript preparation. The University of California has been assigned a patent for the cordance algorithm: software for calculating QEEG cordance is available for academic research collaborations at no cost; additional information is available on our web site, www.QEEG.org.

\section{REFERENCES}

Alvarez JC, Gluck N, Fallet A, Gregoire A, Chevalie JF, Advenier C, Spreux-Varoquaux O (1999): Plasma serotonin level after 1 day of fluoxetine treatment: a biological predictor for antidepressant response? Psychopharmacology (Berl) 143:97-101

Austin MP, Douglas N, Ross M, Murray C, O'Carroll RE, Moffoot A, Ebmeier KP, Goodwin GM (1992): Single photon emission tomography with 99mTc-exametazime in major depression and the pattern of brain activity underlying the psychotic/neurotic continuum. J Affect Disord 26:31-43

Baxter LR Jr, Schwartz JM, Phelps ME, Mazziotta JC, Guze BH, Selin CE, Gerner RH, Sumida RM (1989): Reduction of prefrontal cortex glucose metabolism common to three types of depression. Arch Gen Psychiatry 46:243-250

Beck CH (1995): Acute treatment with antidepressant drugs selectively increases the expression of c-fos in the rat brain. J Psychiatry Neurosci 20:25-32

Bonne O, Krausz Y (1997): Pathophysiological significance of cerebral perfusion abnormalities in major depression-trait or state marker? Eur Neuropsychopharmacol 7:225-233

Bonne O, Krausz Y, Shapira B, Bocher M, Karger H, Gorfine M, Chisin R, Lerner B (1996): Increased cerebral blood flow in depressed patients responding to electroconvulsive therapy. J Nucl Med 37:1075-1080

Brody AL, Saxena S, Silverman DH, Alborzian S, Fairbanks LA, Phelps ME, Huang SC, Wu HM, Maidment K, Baxter LR Jr (1999): Brain metabolic changes in major depressive disorder from pre- to post-treatment with paroxetine. Psychiatry Res 91:127-139

Brody AL, Saxena S, Stoessel P, Gillies LA, Fairbanks LA, Alborzian S, Phelps ME, Huang SC, Wu HM, Ho ML, Ho MK, Au SC, Maidment K, Baxter LR Jr (2001): Regional brain metabolic changes in patients with major depression treated with either paroxetine or interpersonal therapy: preliminary findings. Arch Gen Psychiatry 58:631-640

Cook IA, Leuchter AF (2001): Prefrontal changes and treatment response prediction in depression. Semin Clin Neuropsychiatry 6:113-120

Cook IA, Leuchter AF, Uijtdehaage SHJ, Osato S, Holschneider DH, Abrams M, Rosenberg-Thompson S (1998a): Altered cerebral energy utilization in late life depression. J Affect Disord 49:89-99

Cook IA, O'Hara R, Uijtdehaage SHJ, Mandelkern M, Leuchter AF (1998b): Assessing the accuracy of topographic EEG mapping for determining local brain function. Electroencephalogr Clin Neurophysiol 107:408-414

Cook IA, Leuchter AF, Uijtdehaage SHJ, Abrams M, Anderson-Hanley C, Rosenberg-Thompson S (1999): Neurophysiologic predictors of treatment response to fluoxetine in major depression. Psychiatry Res 85:263-273

Dahmen N, Fehr C, Reuss S, Hiemke C (1997): Stimulation of immediate early gene expression by desipramine in rat brain. Biol Psychiatry 42:317-323 
Drevets WC (1994): Geriatric depression: brain imaging correlates and pharmacologic considerations. J Clin Psychiatry 55(suppl A):71-81; discussion 82, 98-100

Drevets WC, Frank E, Price JC, Kupfer DJ, Holt D, Greer PJ, Huang Y, Gautier C, Mathis C (1999): PET imaging of serotonin 1A receptor binding in depression. Biol Psychiatry 46:1375-1387

Drevets WC, Price JL, Simpson JR Jr, Todd RD, Reich T, Vannier M, Raichle ME (1997): Subgenual prefrontal cortex abnormalities in mood disorders. Nature 386:824-827

Drevets WC, Raichle ME (1992): Neuroanatomical circuits in depression: implications for treatment mechanisms. Psychopharmacol Bull 28:261-274

Dube S, Dobkin JA, Bowler KA, Thase ME, Kupfer DJ (1993): Cerebral perfusion changes with antidepressant repsone in major depression [abstract]. Biol Psychiatry 33:47A

Dunkin JJ, Cook IA, Leuchter AF, Kasl-Godley JE, Abrams M, Betz B, Rosenberg-Thompson S (2000): Executive dysfunction predicts nonresponse to fluoxetine in major depression. J Affect Disord 60:13-23

First M, Spitzer R, Gibbon M, Williams J (1994): Structured Clinical Interview for DSM-IV Axis I Disorders Patient Edition. New York, NY, Biometrics Research Dept., New York State Psychiatric Institute

Frazer A (2001): Serotonergic and noradrenergic reuptake inhibitors: prediction of clinical effects from in vitro potencies. J Clin Psychiatry 62(Suppl 12):16-23

Herrmann WM, Scharer E, Wendt G, Delini-Stula A (1991): Pharmaco-EEG profile of levoprotiline: second example to discuss the predictive value of pharmaco-electroencephalography in early human pharmacological evaluations of psychoactive drugs. Pharmacopsychiatry 24:206-213

Hjorth B (1970): EEG analysis based on time domain properties. Electroencephalogr Clin Neurophysiol 29:306-310

Hjorth B (1975): An on-line transformation of EEG scalp potentials into orthogonal source derivations. Electroencephalogr Clin Neurophysiol 39:526-530

Hurwitz TA, Clark C, Murphy E, Klonoff H, Martin WR, Pate BD (1990): Regional cerebral glucose metabolism in major depressive disorder. Can J Psychiatry 35:684-688

Hyman SE, Nestler EJ (1996): Initiation and adaptation: a paradigm for understanding psychotropic drug action. Am J Psychiatry 153:151-162

Itil TM, Menon GN, Bozak MM, Itil KZ (1984): CNS effects of citalopram, a new serotonin inhibitor antidepressant (a quantitative pharmaco-electroencephalography study). Prog Neuropsychopharmacol Biol Psychiatry 8:397-409

John ER, Prichep LS, Fridman J, Easton P (1988): Neurometrics: computer-assisted differential diagnosis of brain dysfunctions. Science 239:162-169

Kalayam B, Alexopoulos GS (1999): Prefrontal dysfunction and treatment response in geriatric depression. Arch Gen Psychiatry 56:713-718

Katz MM, Koslow SH, Frazer A ((1996): -97) Onset of antidepressant activity: reexamining the structure of depression and multiple actions of drugs. Depress Anxiety 4:257-267

Knott VJ, Telner JI, Lapierre YD, Browne M, Horn ER (1996): Quantitative EEG in the prediction of antidepressant response to imipramine. J Affect Disord 39:175-184

Leuchter AF, Cook IA, Lufkin RB, Dunkin J, Newton TF,
Cummings JL, Mackey JK, Walter DO (1994a): Cordance: a new method of assessment of cerebral perfusion and metabolism using quantitative Electroencephalography. Neuroimage 1:208-219

Leuchter AF, Cook IA, Mena I, Dunkin JJ, Cummings JL, Newton TF, Migneco O, Lufkin RB, Walter DO, Lachenbruch PA (1994b): Assessment of cerebral perfusion and metabolism using Quantitative EEG Cordance. Psychiatry Research: Neuroimaging 55:141-152

Leuchter AF, Cook IA, Newton TF, Dunkin J, Walter DO, Rosenberg-Thompson S, Lachenbruch PA, Weiner $\mathrm{H}$ (1993): Regional differences in brain electrical activity in dementia: use of spectral power and spectral ratio measures. Electroencephalogr Clin Neurophysiol 87:385393

Leuchter AF, Cook IA, Uijtdehaage SHJ, Lufkin RB, Anderson-Hanley C, Abrams M, Rosenberg-Thompson S, O'Hara R, Simon SL, Osato S, Babaie A (1997): Brain structure and function, and the outcomes of treatment for depression. J Clin Psychiatry 58(suppl 16):22-31

Leuchter AF, Uijtdehaage SHJ, Cook IA, O'Hara R, Mandelkern M (1999): Relationship between brain electrical activity and cortical perfusion in normal subjects. Psychiatry Res Neuroimaging 90:125-140

Luthringer R, Dago KT, Patat A, Caille P, Curet O, Durieu G, Rinaudo G, Toussaint M, Granier LA, Macher JP (1996): Pharmacoelectroencephalographic profile of befloxatone, a new reversible MAO-A inhibitor, in healthy subjects. Neuropsychobiology 34:98-105

Martinot JL, Hardy P, Feline A, Huret JD, Mazoyer B, AttarLevy D, Pappata S, Syrota A (1990): Left prefrontal glucose hypometabolism in the depressed state: a confirmation. Am J Psychiatry 147:1313-1317

Muck-Seler D, Jevric-Causevic A, Diksic M (1996): Influence of fluoxetine on regional serotonin synthesis in the rat brain. J Neurochem 67:2434-2442

Newman-Tancredi A, Verriele L, Chaput C, Millan MJ (1996): Down-regulation of rat beta-adrenoceptors by clenbuterol or desipramine does not require chronic treatment: [3H] CGP-12177 binding reveals rapid (24 hour) modulation. Brain Res Bull 41:93-96

Ni YG, Miledi R (1997): Blockage of 5HT2C serotonin receptors by fluoxetine (Prozac). Proc Natl Acad Sci USA 94:2036-2040

Nierenberg AA, McLean NE, Alpert JE, Worthington JJ, Rosenbaum JF, Fava M (1995): Early nonresponse to fluoxetine as a predictor of poor 8-week outcome. Am J Psychiatry 152:1500-1503

Nobler MS, Sackeim HA, Louie J, Prohovnik I, Roose SP, Van Heertum R (1998): Clinical response to antidepressants is associated with reduced frontal CBF in late-life depression. In: Program and Abstracts on New Research: The 151st Annual Meeting of the American Psychiatric Association, Toronto, Ontario, Canada, May 30-June 4, 1998. Washington, DC: The Association;1998:abstract no. NR372.

Nobler MS, Sackeim HA, Prohovnik I, Moeller JR, Mukherjee S, Schnur DB, Prudic J, Devanand DP (1994): Regional cerebral blood flow in mood disorders, III. Treatment and clinical response. Arch Gen Psychiatry 51:884-897

Pitt BR, Weng W, Steve AR, Blakely RD, Reynolds I, Davies 
P (1994): Serotonin increases DNA synthesis in rat proximal and distal pulmonary vascular smooth muscle cells in culture. Am J Physiol 266:L178-L186

Sackeim HA, Nobler MS, Prohovnik I, Moeller JR, Devanand DP, Prudic J (1994): Acute and short-term effects of ECT on regional cerebral blood flow - a marker of treatment adequacy? [abstract]. Neuropsychopharmacology 10:569S

Saletu B, Grunberger J, Linzmayer L, Taeuber K (1982): The pharmacokinetics of nomifensine. Comparison of pharmacokinetics and pharmacodynamics using computer pharmaco-EEG. Int Pharmacopsychiatry 17(Suppl 1):43-72

Saletu B, Grunberger J, Rajna P (1983): Pharmaco-EEG profiles of antidepressants. Pharmacodynamic studies with fluvoxamine. Br J Clin Pharmacol 15(Suppl 3):369S-383S

Saletu B, Grunberger J (1985): Classification and determination of cerebral bioavailability of fluoxetine: pharmacokinetic, pharmaco-EEG, and psychometric analyses. J Clin Psychiatry 46:45-52

Saletu B, Grunberger J, Linzmayer L (1986): On central effects of serotonin re-uptake inhibitors: quantitative EEG and psychometric studies with sertraline and zimelidine. J Neural Transm 67:241-266.

Saletu B, Anderer P, Kinsperger K, Grunberger J (1987): Topographic brain mapping of EEG in neuropsychopharma-
cology-Part II. Clinical applications (pharmaco EEG imaging). Methods Find Exp Clin Pharmacol 9:385-408

Saletu B, Grunberger J (1988): Drug profiling by computed electroencephalography and brain maps, with special consideration of sertraline and its psychometric effects. J Clin Psychiatry 49(Suppl):59-71

Sannita WG (1990): Quantitative EEG in human neuropharmacology. Rationale, history, and recent developments. Acta Neurol (Napoli) 12:389-409

Sannita WG, Ottonello D, Perria B, Rosadini G, Timitilli C (1983): Topographic approaches in human quantitative pharmaco-electroencephalography. Neuropsychobiology 9:66-72

Sulser F (1989): New perspectives on the molecular pharmacology of affective disorders. Eur Arch Psychiatry Neurol Sci 238:231-239

Ulrich G, Haug HJ, Stieglitz RD, Fahndrich E (1988): EEG characteristics of clinically defined on-drug-responders and non-responders-a comparison clomipramine vs. maprotiline. Pharmacopsychiatry 21:367-368

Yatham LN, Liddle PF, Dennie J, Shiah IS, Adam MJ, Lane CJ, Lam RW, Ruth TJ (1999): Decrease in brain serotonin 2 receptor binding in patients with major depression following desipramine treatment: a positron emission tomography study with fluorine-18-labeled setoperone. Arch Gen Psychiatry 56:705-711 